$50 \%$ of those assessed were found to be medium and high risk of malnutrition but it is unclear if they were the patients receiving the correct documentation. Repeat audits could identify this more clearly. We aim to put into place several improvements at ward level to increase compliance. These include; "MUST" calculators, a re-launch of "MUST" screening tools and supporting literature, ward based training. Senior management and ward sisters have been auditing own wards to ensure improvement in compliance with the introduction of ward directed monthly audits. These key clinical indicators of nutrition and hydration have also been based on key recommendations for CQC, DoH and NICE Guidelines.

Competing interests None declared.

\section{PM0-038 A RE-AUDIT TO EVALUATE THE USE OF THE SPINAL NUTRITION SCREENING TOOL IN THE NATIONAL SPINAL INJURIES CENTRE (NSIC) AT STOKE MANDEVILLE HOSPITAL}

doi:10.1136/gutjnl-2012-302514b.38

\begin{abstract}
${ }^{1,2} \mathrm{~S}$ S H Wong, ${ }^{*} \mathrm{~L}$ Spillman, ${ }^{3} \mathrm{~J}$ Doraikannu, ${ }^{3} \mathrm{~A}$ Graham, ${ }^{3} \mathrm{D}$ Green, ${ }^{2} \mathrm{G}$ Grimble, ${ }^{2} \mathrm{~A}$ Forbes. ${ }^{1}$ Nutrition and Dietetics, NATIONAL SPINAL INJURIES CENTRE, Aylesbury; ${ }^{2}$ Centre for Gastroenterology and Clinical Nutrition, University College London, London; ${ }^{3}$ National Spinal Injuries Centre, Stoke Mandeville Hospital, Aylesbury, UK
\end{abstract}

Introduction Malnutrition is common in patients with spinal cord injuries (SCI). ${ }^{1}$ National guidelines ${ }^{2}{ }^{3}$ have set recommended standards for nutrition screening in patients with SCI. An unpublished audit to assess the use of the Spinal Nutrition Screening Tool (SNST) found that in 2009 the use was low.

Methods The present audit aimed to reassess the use of SNST and whether there had been any improvement since 2009. Ninety-three adults (mean age: 51.4 years, SD: 17.3, 17.2\% female) with SCI (53.7\% tetraplegia; $51.6 \%$ complete SCI) were audited in December 2011, on five in-patient wards. Data from individual patient notes were collected by two trained professionals (dietitian and nurse) using a standardised questionnaire.

Results Seventy-one (76.3\%) sets of notes had a SNST form. Sixtynine $(74.2 \%)$ patients had their weight measured on admission, 49 (52.6\%) had their height recorded, and 59 (63.4\%) had their SNST fully completed. At the time of audit, $47.9 \%$ of patients were found to be at risk of undernutrition, $11.5 \%$ had a body mass index $<20 \mathrm{~kg} / \mathrm{m}^{2}$, and $19.4 \%$ were found to have eaten less than half of their last meal. The current audit showed that the use of the SNST has improved significantly: 2009 vs 2011: ward A: 0\% vs $73.3 \%$, p $<0.01$; ward p: $0 \%$ vs $33.3 \%, p=0.01$; ward G: $23.5 \%$ vs $77.3 \%$, $p<0.01$; Ward D: $22 \%$ vs $52 \%, p=0.03$; ward J: $47.6 \%$ vs $81.3 \%$, $p=0.01$; and in the NSIC overall: $24 \%$ vs $63.4 \%,[p<0.001]$.

Conclusion The uptake of nutrition screening appears to have improved in the NSIC but with much still to be achieved. A nutrition steering group has been set up to monitor and evaluate the implementation of nutrition policy on a continuing basis.

Competing interests S Wong: None declared, L Spillman: None declared, J Doraikannu: None declared, A Graham: None declared, D Green: None declared, G Grimble: None declared, A Forbes grant/research support from: National Institute for Health and Research.

\section{REFERENCES}

1. Wong S, et al. Eur J Clin Nutr 2011. doi:10.1038/ejcn.2011.209

2. NICE. Nutrition Support in Adults: Oral Nutrition Support, Enteral Tube Feeding And Parenteral Nutrition. NICE, 2006.

3. Royal College of Physicians. Chronic Spinal Cord Injury: Management of Patients in Acute Hospital Settings: National Guidelines. London: Royal College of Physicians, 2008.

\section{PM0-039 IS NUTRITIONAL RISK ASSOCIATED WITH ADVERSE CLINICAL OUTCOMES SUCH AS LENGTH OF STAY AND MORTALITY IN SPINAL CORD INJURED PATIENTS ADMITTED TO A SPINAL CENTRE?}

doi:10.1136/gutjnl-2012-302514b.39

${ }^{1,2}$ S S H Wong, ${ }^{*}{ }^{3}$ F Derry, ${ }^{3}$ A Jamous, ${ }^{4}$ S Harini, ${ }^{2} \mathrm{G}$ Grimble, ${ }^{2} \mathrm{~A}$ Forbes. ${ }^{1}$ Department of Nutrition and Dietetics, National Spinal Injuries Centre, Aylesbury, UK; ${ }^{2}$ Centre for Gastroenterology and Clinical Nutrition, University College London, London, UK; ${ }^{3}$ National Spinal Injuries Centre, Stoke Mandeville Hospital, Aylesbury, UK; ${ }^{4}$ School of Community and Health Science, City Univeristy, London, UK

Introduction Malnutrition is common in patients with spinal cord injuries (SCI), ${ }^{1}$ but its impact on clinical outcome may be underestimated. A disease specific nutrition screening tool (NST): the Spinal Nutrition Screening Tool (SNST) has been developed for use in patients with SCI but its predictive validity requires further investigation. $^{2}$

Methods A multicentre $(n=4)$, prospective, cross sectional and longitudinal study was therefore performed to evaluate whether undernutrition risk, measured using a simple validated nutrition screening tool (NST): the Spinal Nutrition Screening Tool (SNST), is associated with clinical outcomes such as the duration of in-patient stay (LOS) and 12 month mortality. Multivariate regression analysis was used.

Results One-hundred and fifty SCI patients (aged 18-88, median: $16.9,30.7 \%$ female) were studied in four UK SCI centres (SCIC) between July 2009 and March 2010. The median LOS was 101 days (SD: 94.1 ) and the 12 month mortality rate was $4.7 \% .44 .6 \%$ were at risk of undernutrition and these individuals had a significantly longer LOS [median LOS (SD): 129 (102.1) vs 85 days (84.6); $\mathrm{p}=0.012$ ] and greater 12 -month mortality [ $9.2 \%$ vs $1.4 \% ; \mathrm{p}=0.036$ ]. Multivariate regression identified acute admission and serum albumin level are independent predictors for long hospital LOS.

Conclusion The present study suggests that nutrition risk identified by the SNST score is associated with adverse clinical outcomes. Serum albumin is an independent predictor for an adverse clinical outcome. Nutritional screening on admission and periodic repeating may be helpful in improving clinical outcomes if it is used to influence practice.

Abstract PM0-039 Table 1 Multivariate regression of variables

\begin{tabular}{lccc}
\hline Variables & Un-standardised coefficient (B) & SE & p Value \\
\hline Constant & 197.41 & 72.42 & 0.007 \\
Risk of malnutrition (SNST) & 1.15 & 20.66 & 0.956 \\
Risk of malnutrition (MUST) & 14.27 & 20.11 & 0.478 \\
Type of admission & 81.23 & 15.39 & 0.001 \\
Use of ventilatory support & 37.01 & 26.56 & 0.163 \\
Previous ITU stay & 25.35 & 17.41 & 0.145 \\
Serum albumin & -3.62 & 1.461 & 0.013 \\
Level of SCI & -18.31 & 14.91 & 0.219 \\
Serum total protein & 0.08 & 1.287 & 0.951
\end{tabular}

SNST, Spinal Nutrition Screening Tool; MUST, Malnutrition Universal Screening Tool; ITU, Intensive therapy unit; $\mathrm{SCl}$, spinal cord injury; Adjusted multiple correlation coeffient $\mathrm{R}^{2}$. 0.352 .

Competing interests $S$ Wong grant/research support from: Abbott Nutrition, $F$ Derry: None declared, A Jamous: None declared, S Harini: None declared, G Grimble: None declared, A Forbes: None declared.

\section{REFERENCES}

1. Wong S, et al. Br J Nutr 2011. doi:10.1017/S0007114511006234

2. Wong S, et al. Eur J Clin Nutr 2011. doi:10.1038/ejcn.2011.209 